\title{
Performance Analysis of Blind Adaptive Phase Offset Correction Based on Dispersion Minimization
}

\author{
Wonzoo Chung, William A. Sethares, and C. Richard Johnson, Jr., Fellow, IEEE
}

\begin{abstract}
This paper presents an analysis of the performance and behavior of a blind adaptive carrier phase offset recovery scheme based on dispersion minimization (DM). The algorithm, called the DM-derotator, is a kind of simple equalizer that attempts to correct the carrier phase offset by minimizing the dispersion of the projection of the real part of the (complex) data signal. The recent application of this algorithm to digital broadcasting signals motivates our analysis. This paper classifies the stationary points of the DM-derotator for a variety of source signals including digital vestigial side band (VSB) and quadrature amplitude modulation (QAM) and discusses initialization strategies. The analysis is extended to a variety of situations including $i$ ) its behavior in the presence of intersymbol interference, ii) its behavior when there is statistical dependence between the in-phase and quadrature components, and iii) its tracking ability.
\end{abstract}

Index Terms-Adaptive receiver design, blind adaptive algorithm, carrier phase offset, constant modulus, dispersion minimization.

\section{INTRODUCTION}

I $\mathrm{N}$ modern communication systems, the demand for high data rates over bandlimited channels suggests a preference for blind equalization over training sequence-based equalization schemes. However, in blind equalization schemes, there is, in general, a residual phase ambiguity [14], [20] in the received signal due to the phase distortion of a bandlimited channel or due to carrier phase error [18]. For two-dimensional (2-D) constellations, this phase offset causes a rotation of the equalizer output, which hinders the switch to decision-directed (DD) adaptation. Furthermore, for VSB signals, which have been chosen for digital high-definition TV (HDTV) in the United States [1], the residual phase offset introduces undesirable distortion to the equalizer input signals and can degrade equalizer performance (Section III-B).

Several blind algorithms based on the decision directed approach have been proposed in [15] and [16]. However, reliable decision feedback (which assures convergence of the algorithm) is not always available, especially at the synchronization stage. A blind adaptive carrier phase offset correction algo-

\footnotetext{
Manuscript received January 28, 2002; revised April 28, 2003. This work was supported in part by Fox Digital. The associate editor coordinating the review of this manuscript and approving it for publication was Dr. Alex C. Kot.

W. Chung was with the School of Electrical and Computer Engineering, Cornell University, Ithaca, NY 14853 USA. He is now with Dotcast Inc., Kent, WA 98032 USA (e-mail: wchung@ dotcast.com).

W. A. Sethares is with the Department of Electrical and Computer Engineering, University of Wisconsin, Madison, WI 53706 USA (e-mail: sethares@ece.wisc.edu).

C. R. Johnson, Jr. is with the School of Electrical and Computer Engineering, Cornell University, Ithaca, NY 14853 USA (e-mail: johnson@ece.cornell.edu). Digital Object Identifier 10.1109/TSP.2004.827219
}

rithm, which does not rely on decision feedback, has been mentioned by Bellini in [9, ch. 2] and independently proposed in [3] and [21]. This algorithm minimizes the dispersion of the real component of the received signal in contrast to the MSE-based minimization of decision feedback schemes. The method can be used either before or after equalization, as required by the system. Section II introduces the DM-derotator and analyzes its local minima and local maxima by studying the cost function in an ideal situation. In Section III, the DM-derotator is detailed for quadrature amplitude modulation (QAM) and vestigial side band (VSB) sources, and several examples are given. Section IV studies the behavior of the DM-derotator in less ideal situations, i.e., in the presence of intersymbol interference (ISI), and when there is statistical dependence between the in-phase and quadrature components. In Section V, the tracking ability of the DM-derotator is investigated using a first-order approximation of a linearly time-varying phase offset. The final section concludes.

\section{DM-DEROTATOR}

This section describes the blind phase correction algorithm based on dispersion minimization. The analysis requires certain assumptions under which the cost function of the algorithm can be derived and its stationary points classified. Cases that violate these assumptions are studied in Section IV. A variant of the algorithm, which utilizes a single complex tap, is also considered at the end of this section.

\section{A. DM-Derotator Algorithm}

Suppose that a complex random sequence $\{s\}$ drawn from a finite constellation with known statistical properties suffers from an unknown constant phase offset $\Phi$ in the presence of white complex Gaussian noise $\{w\}$. Consider the measured output

$$
r=s e^{i \Phi}+w
$$

In order to estimate $\Phi$ and directly remove this offset, consider the single tap derotator shown in Fig. 1, where $\phi$ represents an estimate of $\Phi$, and the arrow represents a way of iteratively updating $\phi$. This can be viewed as the problem of equalizing a scalar channel, but conventional blind equalization techniques are not feasible due to their phase ambiguity. Instead, we focus on the projection of the real part (or, equivalently, the imaginary part) of the signal $y$.

In the absence of noise and ISI, and if $\phi$ were exactly equal to $\Phi$, then the projection of $y$ onto the real axis would consist of a collection of points at the (real part of the) symbol values 


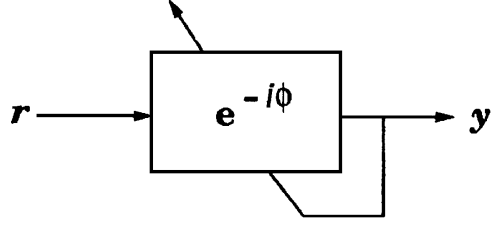

Fig. 1. One tap derotator.

defined by the constellation from which $s$ is drawn. In the presence of noise and ISI, the projection will consist of a number of clusters centered at these symbol values. When $\phi$ is somewhat different from $\Phi$, the clusters widen. Thus, a sensible criterion for estimating $\Phi$ is to try and minimize the dispersion of the projection of the constellation onto the real axis. Formally, consider the cost function, which is named the dispersion minimization derotator (DMD) cost function

$$
J=E\left\{\left(\Re\left(y e^{-i \phi}\right)^{2}-\gamma\right)^{2}\right\}
$$

where $\gamma$ (which will be referred to as the DMD constant) is a real constant, and $\Re(\cdot)$ denotes the real projection operator (i.e., $\Re(a+b i)=a$ ). For many signal constellations, the $\phi$ that minimizes $J(\phi)$ will be equal to $\Phi$, but for some signal constellations, the dispersion can be made even smaller by projecting onto a line other than the real axis. For example, Section IV-B shows that the V29 signal constellation minimizes $J(\phi)$ when $\phi=\Phi+(\pi / 4)$.

Using a stochastic gradient algorithm [8] to minimize (2) gives the DM-derotator algorithm

$$
\phi_{k+1}=\phi_{k}-\mu\left(\Re\left(y e^{-i \phi_{k}}\right)^{2}-\gamma\right) \Re\left(y e^{-i \phi_{k}}\right) \Im\left(y e^{-i \phi_{k}}\right)
$$

which exploits the fact that $(\partial / \partial \phi) \Re\left(y e^{-i \phi_{k}}\right)=\Im\left(y e^{-i \phi_{k}}\right)$, where $\Im(\cdot)$ denotes projection onto the imaginary axis, i.e., $\Im(a+b i)=b$.

This algorithm has been suggested in [9] for QAM, and simulations in [19] indicate good performance, even in the presence of large phase offsets. A variant of this algorithm minimizing the constant modulus cost functions for the real and the imaginary components of the equalizer output simultaneously as well as separately was presented in [12]. The application to VSB signals is considered in [3] and [21]. Together, these studies motivate a more complete analysis.

\section{B. Terminology and Assumptions}

One of the most revealing ways to understand the behavior of an algorithm such as (3) is to study the cost function (or error surface) over which the algorithm evolves. In order to describe the cost function efficiently, some terminology is required.

For a random sequence $\{s\}$, the kurtosis of the source, which is denoted by $\kappa_{s}$, is

$$
\kappa_{s}:=\frac{E\left\{|s|^{4}\right\}}{E\left\{|s|^{2}\right\}^{2}} .
$$

This quantity can be roughly viewed as a measure of the "Gaussian-ness" of the source, and it has been shown to be important in blind identification/equalization schemes based on higher order statistics [2], [5]. The deviation of the kurtosis of a source $\{s\}$ from that of a Gaussian source kurtosis will be denoted by $\eta_{s}$, i.e.,

$$
\eta_{s}:=\kappa_{\text {Gaussian }}-\kappa_{s} .
$$

For a real source, $\eta_{s}=3-\kappa_{s}$. Finally, let $\left\{s_{R}\right\}$ and $\left\{s_{I}\right\}$ denote the real part and the imaginary part of the sequence $\{s\}$, respectively.

$$
s_{R}=\Re(s), \quad s_{I}=\Im(s) .
$$

This paper examines the DM-derotator cost function (2) based on the following assumptions, which are commonly accepted as appropriate for communication systems.

\section{Assumption 1:}

i) $\left\{s_{R}\right\}$ and $\left\{s_{I}\right\}$ are zero-mean and sub-Gaussian, i.e., their kurtoses $\kappa_{s_{R}}$ and $\kappa_{s_{I}}$ are each less than 3 .

ii) The noise $\{w\}$ is complex circular white Gaussian.

iii) The source $\{s\}$ and the noise $\{w\}$ are independent.

\section{Analysis of the Cost Function in an Ideal Scenario}

A variety of constellation sets are used in communication systems to transmit information, and the transmitted signals usually suffer from multipath interference. Depending on the constellation and the multipath channel, the statistical properties of the received signals change, and the DM-derotator cost function may behave differently. Since an exhaustive study of the cost function for each situation would be impractical, this section considers a class of ideal situations that simplify the analysis. Certain nonideal situations of practical significance will be treated separately in the following sections. The assumptions required in this section are as follows.

Assumption 2 (Ideal Situation):

i) The second cross-moment is separable, i.e.,

$$
E\left\{s_{R}^{2} s_{I}^{2}\right\}=E\left\{s_{R}^{2}\right\} E\left\{s_{I}^{2}\right\} .
$$

ii) Most cross-moments vanish

$$
E\left\{s_{R}^{k} s_{I}^{m}\right\}=0, \quad \text { if } k \neq 2, \quad m \neq 2 \quad \text { and } \quad 0<k, m \leq 3 .
$$

iii) The variance of the real and imaginary parts of the signal are the same

$$
E\left\{s_{R}^{2}\right\}=E\left\{s_{I}^{2}\right\} .
$$

In the absence of ISI, most common constellations (such as $n^{2}$-QAM and VSB signals) satisfy the above assumptions (e.g., see Appendix A). Under these assumptions, the DM-derotator cost function has well-defined maxima and minima.

Property (Stationary Points of the DMD): Let $\theta$ denote the parameter error

$$
\theta=\Phi-\phi
$$

between the unknown constant phase $\Phi$ and the DM-derotator parameter $\phi$. Under Assumptions 1 and 2, the DMD cost function (2) has the following stationary points:

$$
\begin{aligned}
\text { Local Minima: } & \theta=0, \pi / 2, \quad \pi, \text { and } \\
\text { Local Maxima: } & \theta= \pm \sin ^{-1} \sqrt{\frac{\eta_{s_{R}}}{\eta_{s_{R}}+\eta_{s_{I}}}} .
\end{aligned}
$$


Proof: The cost function (2) can be rewritten as

$$
J(\theta)=E\left\{\left(\Re\left(s e^{i \theta}+\omega e^{-i \phi}\right)^{2}-\gamma\right)^{2}\right\}
$$

Because $\omega$ is complex circular Gaussian, its statistics are the same as those of $\omega e^{-i \phi}$. Straightforward calculations show that

$$
\begin{aligned}
J(\theta)=m_{2}^{2}\left(\eta_{s_{R}}+\right. & \left.\eta_{s_{I}}\right) \cos ^{4} \theta-2 m_{2}^{2} \eta_{s_{I}} \cos ^{2} \theta+m_{I 4} \\
& -2 \gamma\left(m_{2}+\sigma^{2}\right)+6 m_{2} \sigma^{2}+2 \sigma_{4}+\gamma^{2}
\end{aligned}
$$

where $\sigma^{2}=E\left\{\Re(w)^{2}\right\}, \sigma_{4}=E\left\{\Re(w)^{4}\right\}, m_{2}=E\left\{s_{R}^{2}\right\}=$ $E\left\{s_{I}^{2}\right\}, m_{R 4}=E\left\{s_{R}^{4}\right\}$, and $m_{I 4}=E\left\{s_{I}^{4}\right\}$. The first and the second derivatives of $J$ are given as

$$
\begin{aligned}
\frac{\partial}{\partial \theta} J= & -2 m_{2}^{2}\left(\eta_{s_{R}}+\eta_{s_{I}}\right) \sin 2 \theta\left\{\sin ^{2} \theta-\frac{\eta_{s_{R}}}{\eta_{s_{R}}+\eta_{s_{I}}}\right\} \\
\frac{\partial^{2}}{\partial \theta^{2}} J= & -4 m_{2}^{2}\left(\eta_{s_{R}}+\eta_{s_{I}}\right) \cos 2 \theta\left\{\sin ^{2} \theta-\frac{\eta_{s}}{\eta_{s_{R}}+\eta_{s_{I}}}\right\} \\
& -2 m_{2}^{2}\left(\eta_{s_{R}}+\eta_{s_{I}}\right) \sin ^{2} 2 \theta .
\end{aligned}
$$

Since $\eta_{s_{R}}, \eta_{s_{I}}>0$, at the stationary points $\theta$ with $\sin \theta=$ $\pm \sqrt{\eta_{s_{R}} /\left(\eta_{s_{R}}+\eta_{s_{I}}\right)}$, the second derivative of $J$ is always negative. This shows that they are local maxima and, consequently, that $\{\theta \mid \sin 2 \theta=0\}$ are local minima.

The result of Property 1 reveals several convergence behaviors of this algorithm. First of all, this algorithm inherits a general property of one-dimensional (1-D) gradient systems that have the same number of local minima and local maxima and no saddle points. Usually, stationary points other than local minima in a gradient system significantly slow down the convergence speed, especially in a multimodal system such as CMA where the number of saddle points grows exponentially as the parameter dimension increases [13], [14]. For the 1-D DM-derotator, the cost function can be globally approximated with a piece-wise quartic function partitioned by local maxima (Fig. 2). The convergence speed to the four local minima is slowed only at the vicinity of the four local maxima, and in the rest of the regions, the convergence behavior is governed by the existing analysis on the local convergence of CMA [7], which is similar to the LMS convergence behavior. Since the vicinity of the local maxima is fairy small in the whole parameter space, DMD has an LMS-like local convergence behavior in general. The step size needs to be set to optimize the tradeoff between convergence speed and steady-state error variance.

Second, the DM-derotator is multimodal. The DMD cost function yields a desired local minimum at $\theta=0$ but with a possible ambiguity of $90^{\circ}$. For some sources for which rotationally invariant coding schemes [18] are available (such as $n^{2}$-QAM), this $90^{\circ}$ ambiguity is irrelevant, but for other source signals (such as VSB), it may cause undesirable local minima.

Third, like a CMA equalizer [14], the performance of this algorithm is dependent on the kurtosis of the source. Notice that as $\eta_{s_{R}}, \eta_{s_{I}} \rightarrow 0$ in (7), the cost function becomes flat, which implies that the performance of the DM-derotator degrades as the source approaches Gaussian.

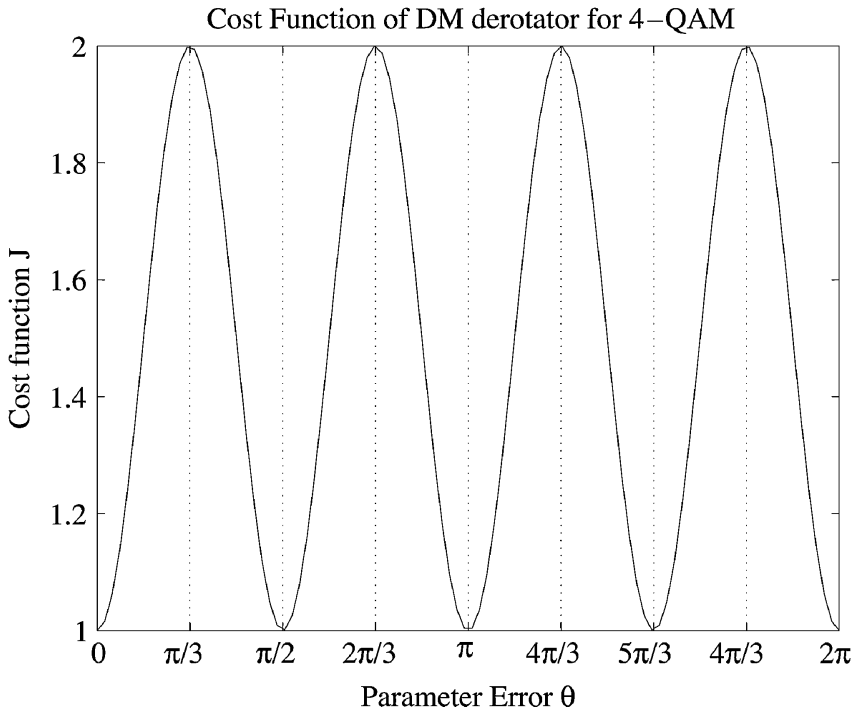

(a)

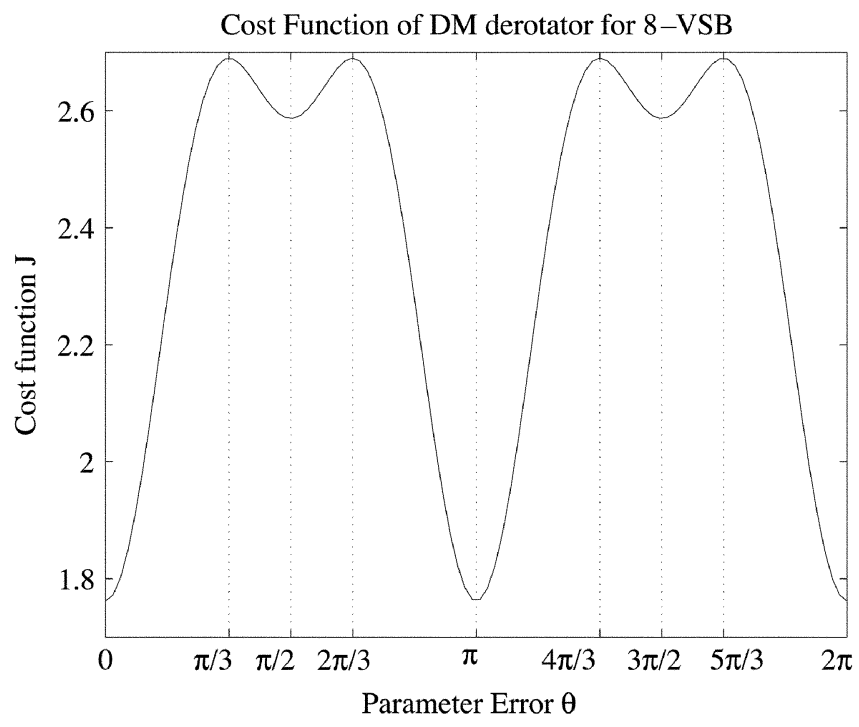

(b)

Fig. 2. Cost function of DM-derotator for QAM and VSB. (a) 4-QAM, (b) 8 -VSB.

Although the 1-D nature of DMD overcomes the slow convergence issues in most blind estimation/adaptation algorithms, the multimodality issue needs to be further discussed, especially for VSB signals. The next sections focus on the DM cost function for VSB and the dynamic changes of DMD when the assumptions for the ideal situation break down. For simplicity of analysis, notice that the DMD constant $\gamma$ contributes only to the constant term in the DMD cost function (7), and hence, it does not change the dynamics of the cost function. Thus, $\gamma$ can be arbitrarily chosen. In practice, $\gamma$ should be chosen to minimize the excess mean squared error of the cost function, but in subsequent sections, $\gamma$ will be set to 0 .

\section{DMD without Norm Constraint}

The DM-derotator, by adjusting only the value of $\phi$ in $e^{i \phi}$, essentially constrains the magnitude of the tap to unity. This 
causes the update term to require the calculation of trigonometric functions, which involves some implementation complexity. This constraint can be relaxed by using a single complex tap update with the cost function

$$
J(\mathrm{f})=E\left\{\left(\Re\left(y f^{*}\right)^{2}-\gamma\right)^{2}\right\}
$$

which leads to the stochastic descent algorithm

$$
\mathrm{f}_{k+1}=\mathrm{f}_{k}+\mu\left(\Re\left(y \mathrm{f}_{k}^{*}\right)^{2}-\gamma\right) \Re\left(y \mathrm{f}_{k}^{*}\right) y .
$$

For the DMD without norm constraint, the DMD constant $\gamma$ must now be adjusted to keep the power gain of the derotator at unity. In conventional CMA equalizers, setting $\gamma=m_{4} / m_{2}$ achieves unity output power [14]. For the DM-derotator, $\gamma$ can be set to either $\gamma=m_{R 4} / m_{2}$ or $\gamma=m_{I 4} / m_{2}$. When $\kappa_{s_{R}} \neq$ $\kappa_{s_{I}}$, i.e., the real and the imaginary parts are not statistically symmetric, the choice of $\gamma$ is roughly equivalent to setting a preference between the real and the imaginary directions. This will be discussed later.

Since the gradient system is identical regardless of the choice of the coordinate system, the cost function can be analyzed in polar coordinates by letting $f=r e^{i \theta}$. Straightforward calculations yield

$$
\begin{aligned}
\frac{\partial}{\partial \theta} J= & -2 m_{2}^{2} r^{4}\left(\eta_{s_{R}}+\eta_{s_{I}}\right) \sin 2 \theta\left\{\sin ^{2} \theta-\frac{\eta_{s_{R}}}{\eta_{s_{R}}+\eta_{s_{I}}}\right\} \\
\frac{\partial}{\partial r} J= & 4 r\left[r ^ { 2 } m _ { 2 } ^ { 2 } \left(\kappa_{s_{R}} \cos ^{4} \theta+\kappa_{s_{I}} \sin ^{4} \theta+\frac{3}{2} \sin ^{2} 2 \theta\right.\right. \\
& \left.\left.+3\left(\frac{\sigma^{4}}{m_{2}^{2}}+2 \frac{\sigma^{2}}{m_{2}}\right)\right)-\gamma\left(m_{2}+\sigma^{2}\right)\right] .
\end{aligned}
$$

Notice that in the angular direction, the cost function has the same local minima and local maxima as in Property 1. In the radial direction, there is a local maximum at $r=0$ and a minima surface given by the equation at the bottom of the page. By letting $\gamma=\kappa_{s_{R}} m_{2}=m_{R 4} / m_{2}$ (provided that the real part is the reference direction), radial values can be specified for each local minimum given in Property 1 . Assuming $m_{2}=1$ (i.e., a normalized source)

$$
r^{2}= \begin{cases}\kappa_{s_{R}} \frac{1+\sigma^{2}}{\kappa_{s_{R}}+3 \sigma^{2}\left(\sigma^{2}+2\right)}, & \text { for } \theta=0, \pi . \\ \kappa_{s_{R}} \frac{1+\sigma^{2}}{\kappa_{s_{I}}+3 \sigma^{2}\left(\sigma^{2}+2\right)}, & \text { for } \theta=\pi / 2, \quad 3 \pi / 2 .\end{cases}
$$

Notice that in the absence of noise, $\left(\sigma^{2}=0\right), r^{2}$ is given by

$$
r^{2}= \begin{cases}1, & \text { for } \theta=0, \pi . \\ \frac{\kappa_{s_{R}}}{\kappa_{s_{I}}}, & \text { for } \theta=\pi / 2, \quad 3 \pi / 2 .\end{cases}
$$

Thus, the gain at $\theta=0$, and at $\theta=\pi$, is unity as desired, whereas the gain at $\theta=\pi / 2$, and at $3 \pi / 2$, is the ratio of the kurtoses between the real and the imaginary parts. The different values of $r$ for different local minima can be used to discern the (possibly undesirable) convergence to the local minima at $\theta=\pi / 2, \theta=3 \pi / 2$ by monitoring $\|\mathrm{f}\|^{2}$, as shown in Section III-B.

\section{EXAMPLES}

\section{A. For $n^{2}$-QAM Signals}

For an $n^{2}$-QAM source, the real and the imaginary components are independent with identical distributions. Thus, in the absence of ISI, the received $n^{2}$-QAM signal satisfies Assumption 2. With the DMD constant $\gamma=0$, the cost function in (7) simplifies to

$$
J=\frac{m_{2}^{2}}{2} \eta_{s} \sin ^{2} 2 \theta+m_{4}+3 \sigma^{4}
$$

which is plotted in Fig. 2(a). Notice that the cost surface is symmetric about $\pi / 2$ due to the statistical homogeneity of the real and the imaginary components of the QAM signal.

\section{B. For Digital VSB Signals}

For digital VSB sources, the real component is an independent and identically distributed (i.i.d.) PAM source, and the complex component is generated by the discrete Hilbert transform of the real component, i.e.,

$$
y[k]=s_{R}[k] \cos \phi+s_{I}[k] \sin \phi,
$$

and

$$
s_{I}[k]=\sum_{n=-\infty}^{\infty} h[n] s_{R}[k-n]
$$

where $h[k]$ is the discrete Hilbert transform filter given explicitly in (27) of Appendix A. The relation between the output sequence $\left\{y_{k}\right\}$ and the source sequence $\left\{s_{k}\right\}$ is represented by a noncasual IIR linear system

$$
\mathrm{y}=(\cos \phi+\sin \phi \mathrm{h}) \star \mathrm{s}
$$

where $\mathrm{s}, \mathrm{y}$, and $\mathrm{h}$ denote the sequence of source, output, and Hilbert transform filters in vector form, $\star$ denotes the convolution operator, and the time indices have been suppressed for notational simplicity. Since $\mathrm{h}^{-1}=-\mathrm{h}$, the phase offset can be corrected by a noncasual IIR filter based on

$$
(\cos \phi+\sin \phi \mathrm{h})^{-1}=\cos \phi-\sin \phi \mathrm{h} .
$$

When ISI is present, channel responses are incorporated in (13) to give

$$
\mathrm{y}=\left(\cos \phi \mathrm{c}_{R}+\sin \phi \mathrm{h} \star c_{I}\right) \star \mathrm{S}
$$

$$
r^{2}=\frac{\gamma\left(1+\sigma^{2} / m_{2}\right)}{m_{2}\left(\kappa_{s_{R}} \cos ^{4} \theta+\kappa_{s_{I}} \sin ^{4} \theta+\frac{3}{2} \sin ^{2} 2 \theta+3\left(\sigma^{4} / m_{2}^{2}+2 \sigma^{2} / m_{2}\right)\right)} .
$$


where $\mathrm{c}_{R}$ and $\mathrm{c}_{I}$ denote the FIR channel responses of the real and the imaginary parts, respectively. Thus, a blind equalization algorithm not only attempts to equalize the channel response but also attempts to compensate for the phase distortion by approximating the phase offset inversion filter (14). While this may seem desirable, in practice, this phase correction property of the equalizer can be a liability. First of all, a large number of taps are utilized to compensate for what is in reality a single parameter distortion. Too many taps will inevitably degrade the steady-state performance of the equalizer and slow convergence. Second, features of the required equalizer impulse response such as sparsity cannot be exploited due to the influence of the Hilbert transform filter. This can be significant in applications such as HDTV, where long equalizers are used [6]. Finally, for a decision feedback equalizer (DFE), the convolution of the Hilbert transform filter with the channel will tend to increase the magnitude of the DFE taps and may result in increased error propagation. Therefore, implementing a blind phase recovery algorithm before equalization is highly desirable for digital VSB signals.

Although the real component $\left\{s_{R}\right\}$ and the imaginary component $\left\{s_{I}\right\}$ of digital VSB signals are statistically dependent, due to the anti-symmetry of the discrete Hilbert transform filter and the i.i.d. property of the real component, they satisfy all conditions in Assumption 2 in the absence of ISI. Furthermore, the kurtosis of the imaginary component is given by

$$
\kappa_{s_{I}}=2+\frac{\kappa_{s_{R}}}{3} .
$$

(A proof of the above statements regarding the statistical properties of the digital VSB signal is given in Appendix A.) Consequently

$$
\sqrt{\frac{\eta_{s_{R}}}{\eta_{s_{R}}+\eta_{s_{I}}}}=\frac{\sqrt{3}}{2} .
$$

This asymmetry of source statistics between the real and the imaginary components induces an asymmetry in the cost function, as shown in Fig. 2(b). For digital VSB signals, $0^{\circ}$ and $180^{\circ}$ are desirable local minima (the resulting output is $s_{R}$ ), whereas $90^{\circ}$ and $270^{\circ}$ are not desirable minima (the resulting output is $s_{I}$ ), which are surrounded by local maxima which occur at $60^{\circ}$, $120^{\circ}, 240^{\circ}$, and $300^{\circ}$ from $\sin \theta= \pm \sqrt{3} / 2$, as described in Property 1 . The convergence to these undesirable local minima can be detected by monitoring the norm of DMD as described in (10). The ratio of the two squared norms at different local minima is given from (10) as

$$
\frac{\|\mathrm{f}\|_{\theta=90^{\circ}}^{2}}{\|\mathrm{f}\|_{\theta=0^{\circ}}^{2}}=\frac{3 \kappa_{s_{R}}+9 \sigma^{2}\left(\sigma^{2}+2\right)}{\kappa_{s_{R}}+6+9 \sigma^{2}\left(\sigma^{2}+2\right)} .
$$

For 8-VSB signals, which are used in the American HDTV standard [1], $\kappa_{s_{R}} \approx 1.762$. For SNR from infinity to $10 \mathrm{~dB}$, the above ratio varies approximately from 0.42 to 0.55 . The squared norm of the undesirable local minima $\|\mathrm{f}\|_{\theta=90^{\circ}}^{2}$ is below 0.42 above $10 \mathrm{~dB}$ SNR, whereas the squared norm of desired local minima $\|\mathrm{f}\|_{\theta=90^{\circ}}^{2}$ stays near 1 and drops to approximately 0.67 at $10 \mathrm{~dB}$ (Fig. 3). Therefore, in most practical situations, it is

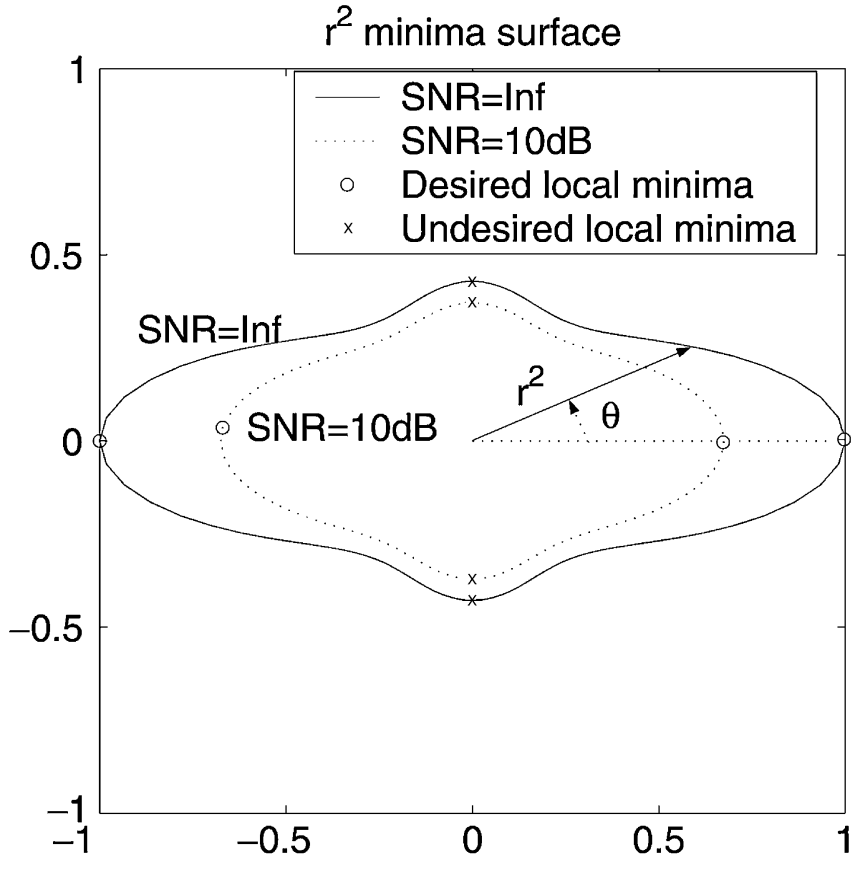

Fig. 3. Squared norm of DMD for VSB signals.

possible to distinguish the desirable from the undesirable local minima. Once detected, an undesirable minimum can be used to direct a reinitialization of the algorithm via a simple rotation.

\section{DMD IN THE PRESENCE OF NONIDEALITIES}

A received signal suffering from ISI, or one generated from a source with an inherent in-phase/quadrature dependence, may violate the conditions in Assumption 2. Since the DM-derotator can be applied after phase invariant blind equalization such as CMA for QAM, ISI may not be a significant obstacle for QAM. However, for VSB signals, ISI can degrade the performance of a DM-derotator. Consequently, this section studies the effect of ISI on the DM-derotator when applied to VSB signals. Other simulated evidence of the behavioral features in this section appears in [4].

\section{A. VSB Under Intersymbol Interference}

In the presence of ISI, VSB signals may not satisfy all the conditions in Assumption 2. For example, let the complex sequence $\{r\}$ arise from a VSB signal as in (15)

$$
r:=\mathrm{c}_{R} \star s_{R}+j \mathrm{c}_{I} \star s_{I} .
$$

Due to the ISI channel, $E\left\{r_{R} r_{I}\right\}$ contains the cross terms $c_{R}[k] c_{I}\left[k^{\prime}\right] E\left\{s_{R}[k] s_{I}\left[k^{\prime}\right]\right\}$. The same argument used in Appendix A shows that

$$
E\left\{s_{R}[k] s_{I}\left[k^{\prime}\right]\right\}=h\left[k-k^{\prime}\right] E\left\{s_{R}^{2}\right\}
$$

which does not vanish unless $k-k^{\prime}$ is even. In this situation, the DMD cost function becomes too complicated to analyze. To succinctly observe the change of the cost function in the presence of ISI, assume that conditions i) and ii) of Assumption 2 still hold but that the third is violated, i.e., $E\left\{r_{R}^{2}\right\} \neq E\left\{r_{I}^{2}\right\}$. 
(Observe also that these assumptions also cover the case of multipath channels that have small $\left\{r_{R}^{k} r_{I}^{m}\right\}(m, l \neq 2)$ terms and $E\left\{r_{R}^{2} r_{I}^{2}\right\} \approx E\left\{r_{R}^{2}\right\} E\left\{r_{I}^{2}\right\}$.)

Let $m_{R_{2}}$ and $m_{I_{2}}$ denote $E\left\{r_{R}^{2}\right\}$ and $E\left\{r_{I}^{2}\right\}$, respectively, and define their ratio as $\rho$,

$$
\rho:=\frac{m_{I_{2}}}{m_{R_{2}}}, \quad \text { i.e., } m_{I_{2}}=\rho m_{R_{2}} \text {. }
$$

Then, the DMD cost function (7) in the presence of ISI can be written as

$$
J_{\mathrm{ISI}}=m_{R_{4}} \cos ^{4} \theta+m_{I_{4}} \sin ^{4} \theta+\frac{3}{2} \rho m_{R_{2}}^{2} \sin ^{2} 2 \theta+C
$$

where $C$ denotes a constant term. The derivative of this cost function is given by

$$
\begin{aligned}
& \frac{\partial}{\partial \theta} J_{\mathrm{ISI}}=-2 m_{R_{2}}^{2}(\left.6 \rho-\kappa_{r_{I}} \rho^{2}-\kappa_{r_{R}}\right) \sin 2 \theta \\
& \times\left\{\sin ^{2} \theta-\frac{3 \rho-\kappa_{r_{R}}}{6 \rho-\kappa_{r_{I}} \rho^{2}-\kappa_{r_{R}}}\right\} .
\end{aligned}
$$

Recall that $\kappa_{r_{R}}$ and $\kappa_{r_{I}}$ are the kurtosis of $c_{R} \star s_{R}$ and $c_{I} \star s_{I}$, respectively. As shown in Appendix A, $\kappa_{r_{R}}$ and $\kappa_{r_{I}}$ are given by

$$
\kappa_{r_{R}}=3-\kappa_{\mathrm{c}_{R}} \eta_{s_{R}}, \quad \kappa_{r_{I}}=3-\kappa_{\mathrm{c}_{I}} \eta_{s_{I}}
$$

where

$$
\kappa_{\mathrm{c}_{R}}:=\frac{\sum c_{R}^{4}[n]}{\left(\sum c_{R}^{2}[n]\right)^{2}}, \quad \kappa_{\mathrm{c}_{I}}:=\frac{\sum \mathrm{c}_{I}^{4}[n]}{\left(\sum c_{I}^{2}[n]\right)^{2}}
$$

From

$$
\sum \frac{c^{4}[n]}{\left(\sum c^{2}[n]\right)^{2}}=\frac{\sum c^{4}[n]}{\sum c^{4}[n]+\sum_{n \neq n^{\prime}} c^{2}[n] c^{2}\left[n^{\prime}\right]} \leq 1
$$

$\kappa_{\mathrm{c}_{R}}$ and $\kappa_{\mathrm{c}_{I}}$ are less than 1 and decrease as the severity of channel multipath increases. Therefore, $\kappa_{r_{R}}$ and $\kappa_{r_{I}}$ are larger than the original $\kappa_{s_{R}}$ and $\kappa_{s_{I}}$ in the presence of an ISI channel.

Notice that the dynamic system described by (19) changes depending on the choice of $\rho$ in (17), and consequently, the cost function (18) may have a different pattern of local minima and local maxima. All the possible stationary points of the cost function (18) can be classified based on $\rho$. (Details are provided in Appendix B.)

i) For $0<\rho<\left(\kappa_{r_{R}}\right) /(3)$

$$
\begin{array}{ll}
\text { Local minima: } & \theta=\pi / 2,3 \pi / 2 . \\
\text { Local maxima: } & \theta=0, \pi .
\end{array}
$$

ii) For $\left(\kappa_{r_{R}}\right) /(3)<\rho<(3) /\left(\kappa_{r_{I}}\right)$

Local minima: $\theta=0, \pi / 2, \pi, 3 \pi / 2$.

Local maxima: $\theta= \pm \sin ^{-1} \sqrt{\frac{3 \rho-\kappa_{r_{R}}}{6 \rho-\kappa_{r_{I}} \rho^{2}-\kappa_{r_{R}}}}$.

iii) For $\rho>(3) /\left(\kappa_{r_{I}}\right)$

Local minima: $\quad \theta=0, \pi$.

Local maxima: $\theta=\pi / 2,3 \pi / 2$.
The only case where the DM-derotator fails to converge to the desired local minima is case $i$ ). The critical bound on $\rho$ for this event is

$$
\rho_{c}:=\frac{\kappa_{r_{R}}}{3}=1-\kappa_{c_{R}} \frac{\eta_{s_{R}}}{3}
$$

which can be close to 1 as the received signals suffer from severe multipath due to decreasing $\kappa_{c_{R}}$. For example, for 8-VSB signals and an ISI-free channel (i.e., $\kappa_{c_{R}}=1$ ), $\rho_{c}=0.5873$. However, multipath interference can increase $\rho_{c}$. For instance, the three-tap channel $\mathrm{c}=[1,0.7,0.5]$ increases $\rho_{c}$ to 0.8225 .

The DM-derotator without norm constraint behaves in a similar fashion, although exact bounds are more complicated. When $\rho$ becomes small, the norm of the (undesirable) local minima in case $i$ ) becomes large in order to compensate for the relatively small power of the imaginary component. Comparing this with the ISI-free case in Section III-B, where the norm of undesirable local minima shrinks, it is clear that monitoring the norm of the DM-derotator parameter is not helpful in the presence of severe ISI when there are significant power differences between the real and the imaginary components. However, even in the undesirable case $\rho<\rho_{c}$, the DM-derotator converges to a solution exactly $90^{\circ}$ off from the correct phase offset. Hence, modest system "intelligence," such as monitoring the cluster variance of the decision device output and introducing $90^{\circ}$ shift to the derotator, can be readily applied to overcome the convergence to undesirable local minima.

\section{B. In-Phase and Quadrature Dependency}

For QAM-type signals, due to the advantageous preprocessing by a blind equalizer, ISI is not a hindrance, but the source sequence may have inherent in-phase and quadrature dependencies. For example, in modified QAM constellations, the source still has identical in-phase and quadrature components but fails to satisfy part ii) of Assumption 2. Instead

$$
E\left\{s_{R}^{2} s_{I}^{2}\right\} \neq E\left\{s_{R}^{2}\right\} E\left\{s_{I}^{2}\right\}=m_{2}^{2} .
$$

In this scenario, the DMD cost function (7) becomes

$$
J_{\text {dep }}=\frac{m_{2}^{2}}{2}\left(\frac{3 E\left\{s_{R}^{2} s_{I}^{2}\right\}}{m_{2}^{2}}-\kappa_{s}\right) \sin ^{2} 2 \theta+C
$$

where $C$ denotes the constant term. When $E\left\{s_{R}^{2} s_{I}^{2}\right\}<m_{2}^{2} \kappa_{\mathrm{s}} / 3$, the sign of the $\sin ^{2} 2 \theta$ term in (21) changes to negative. Thus, the local minima of the cost function is located at undesirable values at $\theta=\pi / 4,3 \pi / 4$, whereas local maxima occur at the desirable values $\theta=0, \pi / 2$.

For example, the V.29 constellation [shown in Fig. 4(a) and used in V.29 modem standard] satisfies this condition. For unit power V.29 sources

$$
\begin{aligned}
& m_{2}=1, \quad m_{4}=4 \cdot \frac{5 \cdot 29}{3^{5}} \approx 2.39, \quad \kappa_{s}=m_{4} \approx 2.39 \\
& E\left\{s_{R}^{2} s_{I}^{2}\right\}=\frac{2^{3} \cdot 41}{3^{6}} \approx 0.45, \quad E\left\{s_{R}^{2} s_{I}^{2}\right\}<\frac{\kappa_{s}}{3} \approx 0.80 .
\end{aligned}
$$

Therefore, when using a constellation such as V.29, the value of $\theta$, which minimizes the cost $J(\theta)$ of (21), is offset from the projection onto the real axis by $45^{\circ}$. Hence, when applying the 


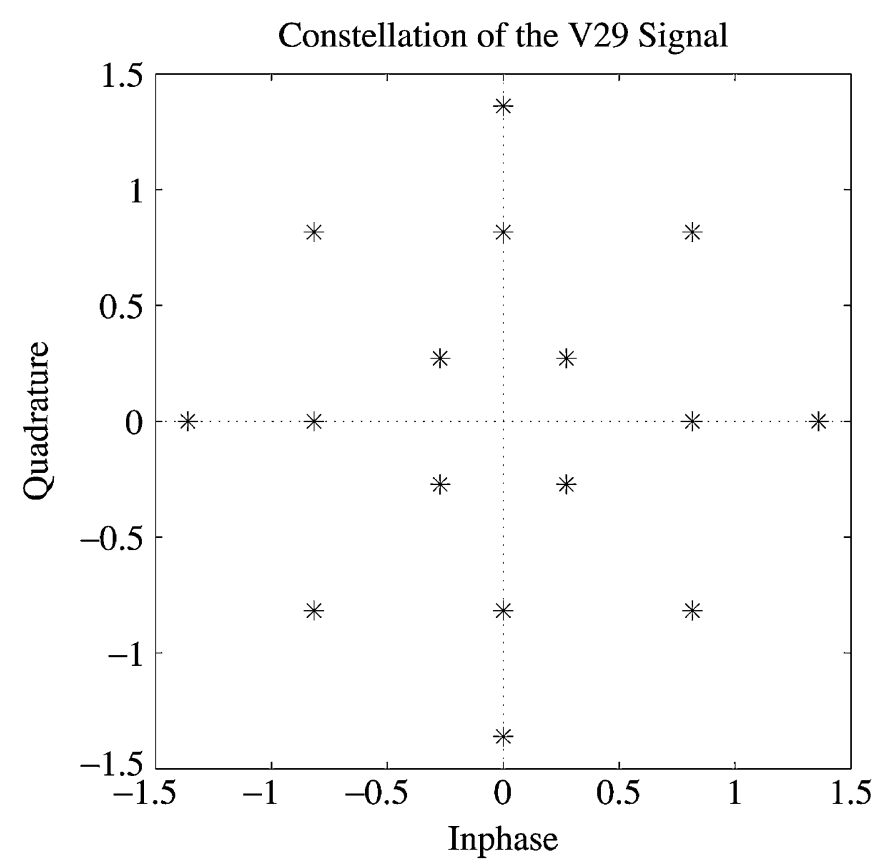

(a)

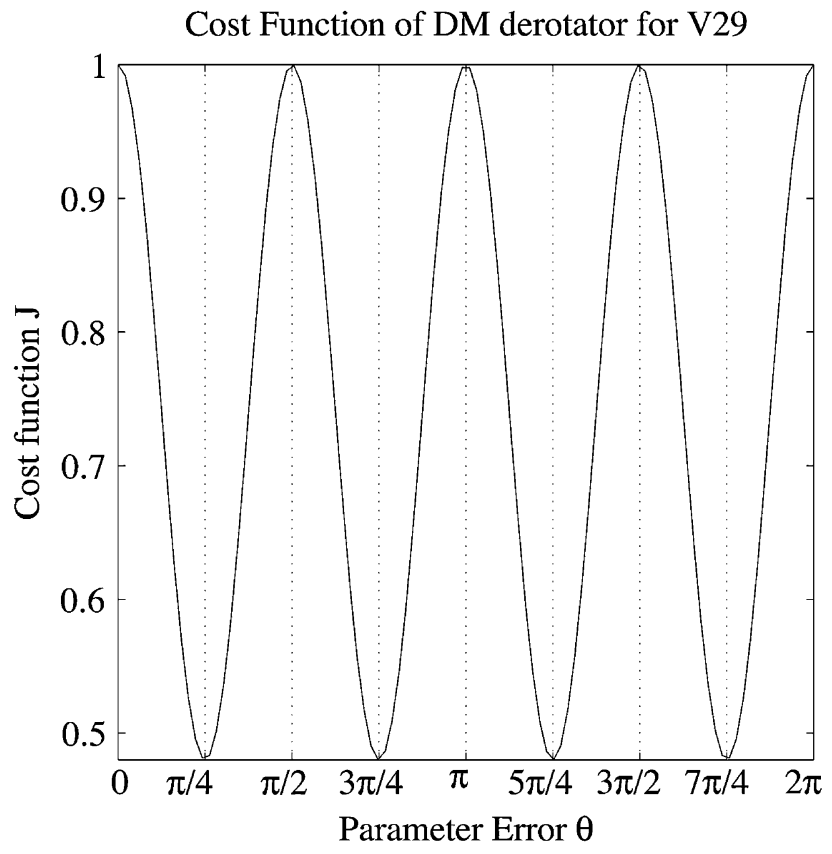

(b)

Fig. 4. DMD cost function for V29 signal. (a) V29 Constellation. (b) Cost function.

DMD to such a constellation, this offset can be accounted for a priori. Alternatively, it is possible to change the sign of the stepsize in the algorithm, effectively searching for the direction that maximizes the dispersion rather than minimizing it.

\section{TRACKING ABILITY OF THE DMD}

In many applications, the phase offset may drift over time due to the frequency offset of the carrier loop. This section investigates the tracking ability of the DM-derotator in the presence of a linear-phase offset under the ideal situations and assumptions of Section II.

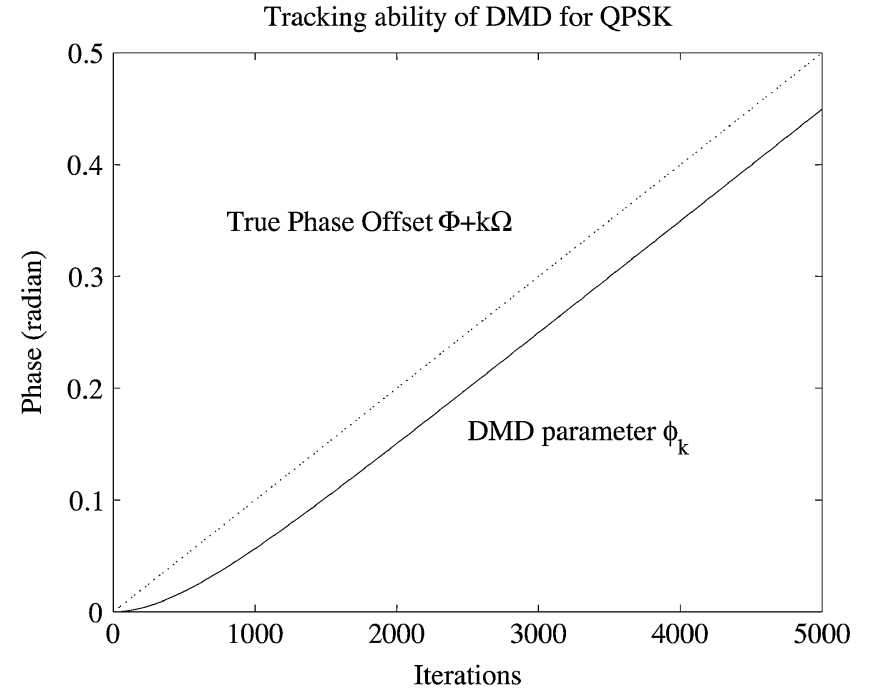

(a)

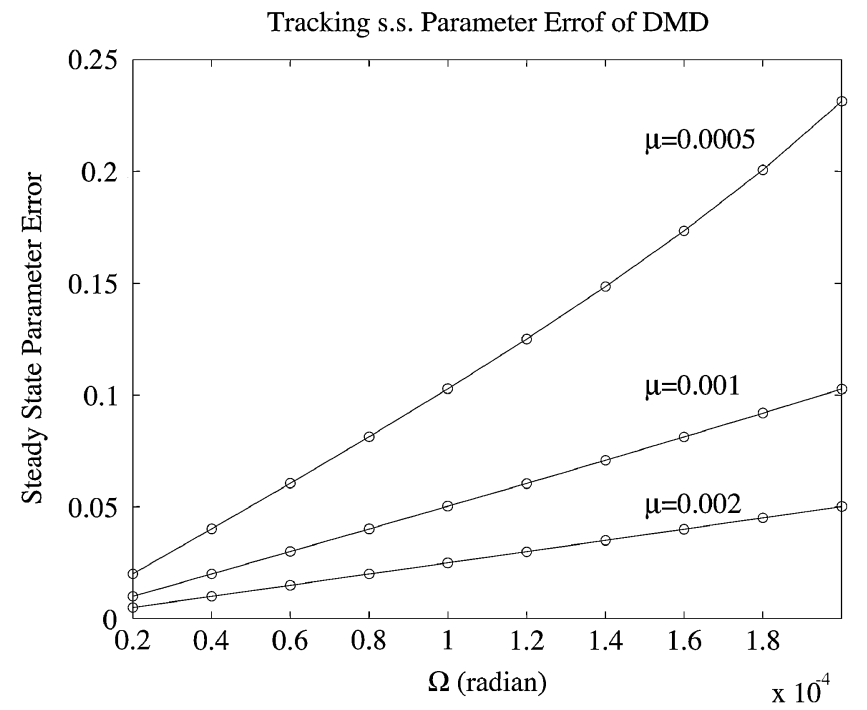

(b)

Fig. 5. Tracking ability of DMD for QPSK. (a) Tracking trace of DMD parameter. (b) $\lim _{k \rightarrow \infty} E\left\{\theta_{k}\right\}$ for various $\Omega$ and $\mu$.

Assume the phase offset is drifting linearly at a rate $\Omega$, i.e., the true phase offset at the $k$ th update is given by $\Phi+k \Omega$. Define

$$
\theta_{k}:=\Phi+k \Omega-\phi_{k}
$$

as the deviation of the estimated parameter of the DM-derotator $\phi_{k}$ from the true phase offset. From the update (3) (again without loss of generality, let $\gamma=0$ )

$$
\theta_{k+1}=\theta_{k}+\Omega-\mu \Re\left(s e^{i \theta_{k}}\right)^{2} \Re\left(s e^{i \theta_{k}}\right) \Im\left(s e^{i \theta_{k}}\right) .
$$

By taking the ensemble average of the above nonlinear dynamic system under Assumptions 1 and 2

$$
\begin{aligned}
E\left\{\theta_{k+1}\right\}= & E\left\{\theta_{k}\right\}+\Omega+\mu \frac{m_{2}^{2}}{2} E \\
& \times\left\{\sin 2 \theta_{k}\left[\left(\eta_{s_{R}}+\eta_{s_{I}}\right) \sin ^{2} \theta_{k}-\eta_{s_{R}}\right]\right\} .
\end{aligned}
$$

Assume a steady state of the above system as illustrated in Fig. 5(a) so that $\lim _{k \rightarrow \infty} E\left(\theta_{k+1}\right)=\lim _{k \rightarrow \infty} E\left(\theta_{k}\right)$, and 
further assume that $\Omega$ is small enough to validate the first-order approximation in the expectation term in (24). Then

$$
\begin{aligned}
\lim _{k \rightarrow \infty} E\left\{\sin 2 \theta_{k}\left[\left(\eta_{s_{R}}+\eta_{s_{I}}\right) \sin ^{2} \theta_{k}-\eta_{s_{R}}\right]\right\} & \\
& \approx-2 \eta_{s_{R}} \lim _{\kappa \rightarrow \infty} E\left\{\theta_{k}\right\} .
\end{aligned}
$$

Using this first-order approximation gives

$$
\lim _{k \rightarrow \infty} E\left(\theta_{k}\right) \approx \frac{\Omega}{\mu m_{2}^{2} \eta_{s_{R}}} .
$$

For example, for a unit power QPSK source $\left(\eta_{s}=2\right.$ and $m_{2}^{2}=$ 1 ), $\Omega=1 \times 10^{-4}$ (radian), and $\mu=1 \times 10^{-3}$, the steady-state parameter error $\lim _{k \rightarrow \infty} E\left(\theta_{k}\right)$, given by the above formula, is 0.05 . This agrees well with simulation results for small $\Omega$ [Fig. 5(a)]. Fig. 5(b) shows the steady-state parameter errors for various $\Omega$ and $\mu$. For $\mu=0.001$ and 0.002 , the simulation results of the steady-state parameter error agree with the approximation values given in (26) for $\Omega$ in the range between $2 \times 10^{-5}$ and $2 \times 10^{-4}$. However, for $\mu=0.0005$, the steady-state parameter error deviates from the approximation values for large $\Omega$, which shows the limit of the validity of the "small $\Omega$ assumption" in (26).

\section{CONCLUSION}

We have presented analysis of a blind adaptive phase correction algorithm based on dispersion minimization. We have derived a general cost function under acceptable assumptions in the absence of ISI and confirmed that the DM-derotator works as expected for QAM and VSB sources. We have extended the analysis to a number of situations of practical significance, to the presence of ISI, to nonconventional source signals, and to an investigation of the tracking ability of the algorithm.

\section{APPENDIX A}

\section{Statistical Properties of Digital VSB}

For a digital VSB signal

$$
s_{I}[k]=\sum_{n=-\infty}^{\infty} h[n] s_{R}[k-n]
$$

where $h$ is the discrete Hilbert transform filter given by [17]

$$
h[n]= \begin{cases}2 \frac{\sin ^{2}(\pi n / 2)}{\pi n}, & \text { for } n \neq 0 \\ 0, & \text { for } n=0\end{cases}
$$

First

$$
\begin{aligned}
E\left\{s_{R}^{2}[k] s_{I}^{2}[k]\right\} & =E\left\{s_{R}^{2}[k] \sum_{n} h[n]^{2} s_{R}^{2}[k-n]\right\} \\
& =\sum_{n \neq 0} h[n]^{2} E\left\{s_{R}^{2}[k]\right\} E\left\{s_{R}^{2}[k-n]\right\} \\
& =E\left\{s_{R}^{2}[k]\right\} E\left\{s_{R}^{2}[k]\right\}\|\mathrm{h}\|^{2} \\
& =E\left\{s_{R}^{2}[k]\right\} E\left\{s_{I}^{2}[k]\right\}
\end{aligned}
$$

which establishes Assumption 2(i). The derivation has used the i.i.d property of $\left\{s_{R}\right\}, h[0]=0$, and $\|h\|=1$. Furthermore

$$
\begin{aligned}
E\left\{s_{R}[k] s_{I}[k]\right\}=\sum_{n \neq 0} h[n] E\left\{s_{R}[k] s_{R}[k\right. & -n]\} \\
& +h[0] E\left\{s_{R}[k]^{2}\right\}=0 .
\end{aligned}
$$

Similarly, it is not difficult to check that

$$
E\left\{s_{R}^{p}\left[[k] s_{I}^{q}[k]\right\}=0, \quad E\left\{s_{R}^{2}[k]\right\}=E\left\{s_{I}^{2}[k]\right\}\right.
$$

for $1 \leq p, q \leq 3$, and $p \neq q$, which satisfies Assumption 2 (ii) and (iii).

In order to calculate the kurtosis of $\left\{s_{I}\right\}$, first, consider a given channel $\mathrm{c}$ and source $\{s\}$. The kurtosis of $\{\mathrm{c} \star s\}$ is given by

$$
\kappa_{\mathrm{c} \star \mathrm{s}}=3-\kappa_{c} \eta_{s}
$$

where $\kappa_{c}=\left(\sum_{n} \mathrm{c}^{4}[n]\right) /\|\mathrm{c}\|^{4}$. The derivation is as follows:

$$
\begin{aligned}
E\left\{(\mathrm{c} \star \mathrm{s}[k])^{4}\right\}= & E\left\{\left(\sum_{n} \mathrm{c}[n] \mathrm{s}[k-n]\right)^{4}\right\} \\
= & \sum \mathrm{c}^{4}[n] E\left\{\mathrm{~s}^{4}[k-n]\right\} \\
& +3 \sum_{n \neq n^{\prime}} \mathrm{c}^{2}[n] \mathrm{c}^{2}\left[n^{\prime}\right] E\left\{\mathrm{~s}^{2}[k-n]\right\} E\left\{\mathrm{~s}^{2}\left[k-n^{\prime}\right]\right\} \\
= & m_{4} \sum \mathrm{c}^{4}[n]+3 m_{2}^{2}\left(\|\mathrm{c}\|^{4}-\sum \mathrm{c}^{4}[n]\right) \\
= & 3 m_{2}^{2}\|\mathrm{c}\|^{4}-\left(m_{2}^{2}-m_{4}\right) \sum \mathrm{c}[n]^{4}
\end{aligned}
$$

where we have used the algebraic fact that

$$
\sum_{n \neq n^{\prime}} c^{2}[n] c^{2}\left[n^{\prime}\right]=\|c\|^{4}-\sum c^{4}[n] .
$$

On the other hand

$$
E\left\{(\mathrm{c} \star \mathrm{s}[k])^{2}\right\}=m_{2}\|\mathrm{c}\|^{2} .
$$

Therefore

$$
\begin{aligned}
\kappa_{\mathrm{c} \star \mathrm{s}} & =\frac{3 m_{2}^{2}\|c\|^{4}-\left(3 m_{2}^{2}-m_{4}\right) \sum c[n]^{4}}{m_{2}^{2}\|\mathrm{c}\|^{4}} \\
& =3-\kappa_{\mathrm{c}} \eta_{s} .
\end{aligned}
$$

Using this formula and the fact that for the discrete Hilbert transform filter, $\|h\|^{2}=1$, and

$$
\sum_{n=\infty}^{\infty} h[n]^{4}=\sum_{n=1}^{\infty} \frac{2 \cdot 2^{4}}{\pi^{4}(2 n-1)^{4}}=\frac{1}{3}
$$

which uses the summation formula [11],

$$
\sum_{n=1}^{\infty} \frac{1}{(2 n-1)^{4}}=\frac{\pi^{4}}{3 \cdot 2^{5}}
$$

we finally have

$$
\kappa_{s_{I}}=3-\frac{1}{3}\left(3-\kappa_{s_{R}}\right)=2+\frac{\kappa_{s_{R}}}{3} .
$$


TABLE I

\begin{tabular}{c|c|c}
\hline \multicolumn{2}{c|}{ Cases } & Range of $\rho$ \\
\hline \multirow{3}{*}{ 1) $Q_{1}<0$} & $Q_{2}>0$ & $(3-\sqrt{D}) / \kappa_{r_{I}}<\rho<\kappa_{r_{R}} / 3^{*}$ \\
\cline { 2 - 3 } & $Q_{2}<0$ & $(3+\sqrt{D}) / \kappa_{r_{I}}<\rho$ \\
\hline \multirow{3}{*}{$2) 0<Q_{1}<1$} & $Q_{2}>0$ & $\kappa_{r_{R}} / 3<\rho<\kappa_{r_{I}} / 3$ \\
\cline { 2 - 3 } & $Q_{2}<0$ & $\phi$ \\
\hline \multirow{3}{*}{ 3) $Q_{1}>1$} & $Q_{2}>0$ & $3 / \kappa_{r_{I}}<\rho<(3+\sqrt{D}) / \kappa_{r_{I}}$ \\
\cline { 2 - 3 } & $Q_{2}<0$ & $0<\rho<(3-\sqrt{D}) / \kappa_{r_{I}}$ \\
\hline \multirow{2}{*}{$*:(3-\sqrt{D}) / k_{r_{I}}<\kappa_{r_{R}} / 3$ always holds. }
\end{tabular}

APPENDIX B

CHANGE OF THE DMD COST FUNCTION FOR VSB IN THE PRESENCE OF ISI

The derivation of the DMD cost function in the presence of ISI yields [from (19)]

$$
\begin{aligned}
\frac{\partial J_{\mathrm{ISI}}}{\partial \theta}=-2 m_{R}^{2}\left(6 \rho-\kappa_{r_{I}} \rho^{2}-\kappa_{r_{R}}\right) \sin 2 \theta & \\
\times & \times\left\{\sin ^{2} \theta-\frac{3 \rho-\kappa_{r_{R}}}{6 \rho-\kappa_{r_{I}} \rho^{2}-\kappa_{r_{R}}}\right\} .
\end{aligned}
$$

Define the following two terms which can change the dynamics of the cost function:

$$
\begin{aligned}
Q_{1} & =\frac{3 \rho-\kappa_{r_{R}}}{6 \rho-\kappa_{r_{I}} \rho^{2}-\kappa_{r_{R}}}, \\
Q_{2} & =6 \rho-\kappa_{r_{I}} \rho^{2}-\kappa_{r_{R}} .
\end{aligned}
$$

Now, we tabulate the ranges of $\rho$ that change the signs of $Q_{1}$ and $Q_{2}$. After solving a series of quadratic equations, we summarize in Table I, where $D=9-\kappa_{r_{R}} \kappa_{r_{I}}$. From the second derivative

$$
\frac{\partial^{2}}{\partial \theta^{2}} J_{\text {ISI }}=-4 m_{2}^{2} Q_{2} \cos 2 \theta\left\{\sin ^{2} \theta-Q_{1}\right\}-2 m_{2}^{2} Q_{2} \sin ^{2} 2 \theta
$$

we can observe that in case 2) for the stationary points $\left\{\theta \mid \sin ^{2} \theta=Q_{1}\right\}$

$$
\left.\frac{\partial^{2}}{\partial \theta^{2}} J_{\text {ISI I }}\right|_{\theta= \pm \sin ^{-1} \sqrt{Q_{2}}}=-2 m_{2}^{2} Q_{2} \sin ^{2}\left(2 \sin ^{-1} \sqrt{Q_{1}}\right)<0
$$

and therefore, $\left\{\theta \mid \sin ^{2} \theta=Q_{1}\right\}$ are local maxima. For a 1-D cost function, this implies that any stationary point located between two consecutive elements in $\left\{\theta \mid \sin ^{2} \theta=Q_{1}\right\}$ must be a local minimum. Therefore, $\{\theta \mid \sin 2 \theta=0\}$ are local minima for case 2). For cases 1a) and $3 \mathrm{~b}$ ), the cost function behaves like a nonuniformly distorted $\cos 2 \theta$ and has local maxima at $\theta=$ $0, \pi$ and local minima at $\theta=\pi / 2,3 \pi / 2$. Similarly, for cases $1 \mathrm{~b}$ ) and 3a) the cost function yields local maxima at $\theta=\pi / 2,3 \pi / 2$ and local minima at $\theta=0, \pi$.

\section{REFERENCES}

[1] ATSC Digital Television Standard (Doc. A/53), http://www.atsc.org, Mar. 2000. Advanced Television Syst. Committee.

[2] A. Benveniste, M. Goursat, and G. Ruget, "Robust identification of a nonminimum phase system: Blind adjustment of a linear equalizer in data communications," IEEE Trans. Automat. Contr., vol. AC-25, pp. 385-399, June 1980.

[3] W. Chung, W. A. Sethares, and C. R. Johnson Jr., "Blind adaptive phase offset correction," in Proc. IEEE GLOBECOM, San Francisco, CA, Nov. 2000 .

[4] W. Chung, "Blind Parameter Estimation for Data Acquisition in Digital Communication Systems," Ph.D. dissertation, Sch. Elect. Comput. Eng., Cornell Univ., Ithaca, NY, Aug. 2002.

[5] D. L. Donoho, "On minimum entropy deconvolution," in Applied Time Series Analysis II, D. Findley, Ed. New York: Academic, 1981, pp. 565-608.

[6] T. J. Endres, R. A. Casas, S. N. Hulyalkar, and C. H. Strolle, "On sparse equalization using mean-square-error and constant modulus criteria," in Proc. Conf. Inform. Sci. Syst.. Princeton, NJ, Mar. 2000.

[7] Z. Gu and W. A. Sethares, "A geometrical view of blind equalization," in Proc. IEEE Int. Conf. Acoustics, Speech, Signal Processing, vol. 3, Minneapolis, MN, Apr. 27-30, 1993, pp. 551-554.

[8] J. R. Treichler, C. R. Johnson Jr., and M. G. Larimore, Theory and Design of Adaptive Filters. Englewood Cliffs, NJ: Prentice-Hall, 2001.

[9] S. Haykin, Blind Deconvolution. Englewood Cliffs, NJ: Prentice-Hall, 1994.

[10] - Communication Systems. New York: Wiley, 1983.

[11] L.B.W. Jolley, Summation of Series. New York, NY: Dover, 1961.

[12] K. N. Oh and Y. O. Chin, "Modified constant modulus algorithm: Blind equalization and carrier phase recovery algorithm," in Proc. IEEE Int. Conf. Commun., vol. 1, 1995, pp. 498-502.

[13] S. Lambotharan, J. Chambers, and C. R. Johnson Jr., "Attractions of saddles and slow convergence in CMA adaptation," Signal Process., vol. 59, pp. 335-340, June 1997.

[14] C. R. Johnson, Jr., P. B. Schniter, T. S. Endres, J. M. Behm, D. R. Brown, and R. A. Casas, "Blind equalization using the constant modulus criterion: A review," Proc. IEEE, vol. 86, pp. 1927-1950, Oct. 1998.

[15] O. Macchi, Adaptive Processing. New York: Wiley, 1995.

[16] H. Meyr, M. Moeneclaey, and S. A. Fechtel, Digital Communication Receivers. New York: Wiley, 1998.

[17] A. V. Oppenheim and R. W. Shafer, Discrete-Time Signal Processing. Englewood Cliffs, NJ: Prentice-Hall, 1999.

[18] J. G. Proakis, Digital Communications, 2nd ed. New York, NY: McGraw-Hill, 1989.

[19] J. J. Shynk, C. K. Chan, and R. P. Gooch, "Comparative performance study of several blind equalization algorithms," in Proc. SPIE, San Diego, CA, 1991.

[20] L. Tong, G. Xu, and T. Kaliath, "Blind channel identification and equalization using second-order statistics: A time-domain approach," IEEE Trans. Inform. Theory, vol. 40, pp. 340-349, Mar. 1994.

[21] A. Touzni, R. A. Casas, T. J. Endres, C. H. Strolle, and S. Hulyalkar, "Phase recovery based on minimization of single-axis constant modulus criterion: Performance analysis," in Proc. Conf. Inform. Sci. Syst., Baltimore, MD, Mar. 2001.

[22] J. R. Treichler and B. G. Agee, "A new approach to multipath correction of constant modulus signals," IEEE Trans. Acoust., Speech, Signal Processing, vol. ASSP-31, pp. 459-472, Apr. 1983.

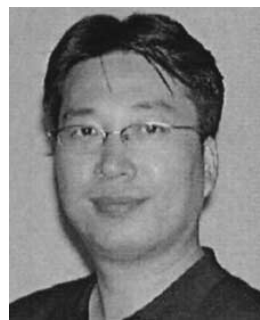

Wonzoo Chung received the B.A. degree in mathematics from Korea University, Seoul, Korea, and the M.S. and Ph.D. degrees in electrical engineering from Cornell University, Ithaca, NY.

$\mathrm{He}$ is currently with Dotcast, Inc., Kent, WA, as a Systems Engineer, working on research and development of signal processing technologies for the digital data broadcasting system. His research interests include blind and adaptive aspects of digital signal processing for telecommunication systems. 


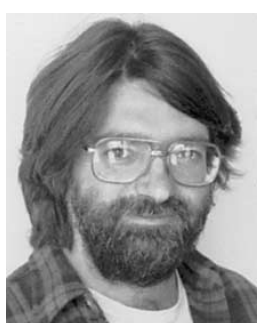

William A. Sethares received the B.A. degree in mathematics from Brandeis University, Waltham, MA and the M.S. and Ph.D. degrees in electrical engineering from Cornell University, Ithaca, NY.

$\mathrm{He}$ has worked at the Raytheon Company, Waltham, as a Systems Engineer and is currently a Professor with the Department of Electrical and Computer Engineering, University of Wisconsin, Madison. His research interests include adaptation and learning in signal processing, communications, and acoustics. He is is author of Tuning, Timbre, Spectrum, Scale (New York: Springer 1998) and of Telecommunication Breakdown (Englewood Cliffs, NJ: Prentice-Hall 2003).

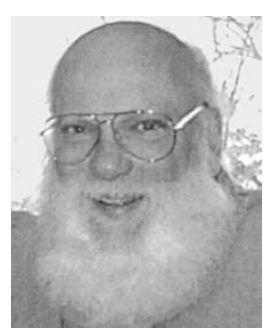

C. Richard Johnson, Jr. (F'89) was born in Macon, GA, in 1950. He received the Ph.D. degree in electrical engineering with minors in engineering-economic systems and art history from Stanford University, Stanford, CA, in 1977.

$\mathrm{He}$ is currently a Professor of electrical and computer engineering and a member of the Graduate Field of Applied Mathematics at Cornell University, Ithaca, NY. Since 1990, he has held visiting appointments at Stanford University; the University of California-Berkeley; Chalmers University of Technology, Gothenburg, Sweden; the Technical University of Vienna, Vienna, Austria; National Polytechnic Institute of Grenoble, Grenoble, France; and the Australian National University, Canberra, Australia. During that period, his primary research interest has been blind adaptive fractionally spaced linear and decision feedback equalization for intersymbol and structured multiuser interference removal from single and multicarrier communication systems. The current broadband adaptive receiver design project of his research group at Cornell is described at http://bard.ece.Cornell.edu/. The group's activity is currently supported by the National Science Foundation, Applied Signal Technology, and Texas Instruments.

Dr. Johnson was elected a Fellow of the IEEE "for contributions to adaptive parameter estimation theory with applications in digital control and signal processing" in 1989. 